\title{
Stimulus intensity and familiarization effects on simple RT performance in children'
}

\section{FRANK D. MILLER and GENE H. MOFFAT, ${ }^{2}$ University of South Dakota, Vermillion, South Dakota 57069}

Young children, assigned to a High, Medium, or Low stimulus intensity group, were given 71 simple reaction time trials. On the first 30 trials, a familiarization stimulus was presented. For the remaining 41 trials, Ss responded on each trial to the onset of either the familiarized or a nonfamiliarized stimulus. Stimulus intensity was not an effective variable. A stimulus familiarization effect was obtained, whereas a change effect was not. The latter result is inconsistent with a stimulus interpretation of the change effect.

A paradigm used to examine the effects of familiarization on response speeds in children involves the repeated presentation of a stimulus to which $S$ attends or also responds during a familiarization phase. This phase is followed by a motor task in which $S$ responds to the onset of either the familiarized stimulus (FS) or a nonfamiliarized stimulus (NS) not presented in the familiarization phase. A stimulus familiarization effect (SFE), indicated by faster response speeds to the NS than to the FS, has been consistently demonstrated in both single and differential response tasks (e.g., Bogartz \& Witte, 1966; Cantor \& Cantor, 1964, 1965, 1966). Cantor \& Cantor (1965) present a habituation hypothesis for the SFE. Attending responses may habituate to the FS during the familiarization phase. Consequently, in the motor task phase Ss may show greater habituation to the FS, and hence slower speeds to the FS than to the NS. Thompson \& Spencer (1966) maintain that the weaker the stimulus, the more rapid and/or more pronounced is habituation for most types of responses, whereas intense stimuli may yield no significant habituation.

One purpose of the present study was to investigate the effects of stimulus intensity on response speeds in both the familiarization and two-stimulus reaction phases. The above hypotheses in combination suggest that in the familiarization phase, the decrement in response speed over trials would be least for the High intensity group, more for the Medium intensity group, and most for the Low intensity group. In the two-stimulus reaction phase, the differences in speed of responding to the FS and NS would be smallest for the High intensity group, intermediate for the Medium intensity group, and largest for the Low intensity group. These effects would be evidenced in a Stimulus Intensity by Trial Block interaction in a familiarization phase analysis and a SFE by Intensity interaction in a two-stimulus RT phase analysis.

The change effect (CE) was of additional interest. The CE is characterized by faster response speeds on trials involving a change of stimulus from the previous trial (i.e., NS following FS and FS following NS) than on trials involving no change of stimulus (i.e., NS following NS and FS following FS). Positive evidence for the $\mathrm{CE}$ has been obtained when a differential motor response is required and when, in addition, the stimulus sequences have involved predominantly more change than nonchange trials (Bogartz \& Witte, 1966, Experiment 2; Cantor \& Cantor, 1965, 1966). The CE has not been demonstrated in either a simple RT (Bogartz \& Witte, 1966, Experiment 1) or a lever pulling task (e.g., Witte, 1967), in which $S$ is required to make the same motor response to the NS and FS. The second purpose of this study was to further investigate whether a CE could be obtained in a simple RT task.
METHOD
The Ss were 20 four-year-old children enrolled in the University of South Dakota Nursery School and 43 five- and six-year-old children from Austin Elementary School, Vermillion, South Dakota. Each $S$ was tested in a dimly illuminated room using a single-aperture, single-response button apparatus. The Ss were assigned to High, Medium, or Low light intensity groups: $17.00,3.08$, and $1.60 \mathrm{ft}-\mathrm{L}$, respectively. For half the $S s$ in each group, the familiarization light was red; for the remaining $S s$, it was green. Each intensity-color subgroup was equated for age and consisted of six boys and six girls. After $\mathrm{S}$ was seated, he was instructed to watch carefully for a light to come on and to turn it off as quickly as possible by moving his hand from a start pad to the response button and depressing it. No ready signal was provided. Stimulus duration was $3 \mathrm{sec}$ on all trials and the intertrial interval (i.e., time from stimulus offset to onset of next stimulus) was 3,4 , or $5 \mathrm{sec}$ according to a random order common to all Ss. Stimulus duration and intertrial interval were controlled by a Gerbrands $2 \mathrm{~mm} / \mathrm{sec}$ Tape Interval Timer.

In the familiarization phase, $S s$ responded to 30 presentations of the familiarization stimulus according to their subgroup specifications. Forty-one simple RT trials were then administered without interruption. Four different sequences of the NS and FS were used. Each one-fourth of the Ss familiarized on red were given one of the four different sequences; Ss familiarized on green were treated in an identical manner. Two sequences contained $20 \mathrm{NS}$ and 21 FS presentations, while the remaining sequences consisted of 21 NS and 20 FS presentations. All sequences involved $50 \%$ alternation and $50 \%$ repetition of the NS and FS. Taking into account the type of stimulus occurring on the immediately preceding trial, this procedure resulted in 10 occurrences of each of the four possible sequential events (i.e., FS-NS, NS-FS, NS-NS, FS-FS). Time from stimulus onset to button depression was recorded to the nearest $.01 \mathrm{sec}$.

\section{RESULTS}

Response latencies on all trials were made reciprocal to produce speed scores. The mean speeds for the six trial blocks were: $1.196,1.445,1.515,1.439,1.417$, and 1.322 , respectively. A Type III analysis of variance (Lindquist, 1953) performed on mean trial block speeds in the familiarization phase indicated that, while stimulus intensity $(F<1)$ and color $(F<1)$ were not significant as between-Ss variables, the within-Ss trial-block effect was significant $(F=4.03$ $\mathrm{df}=5 / 330, \mathrm{p}<.01)$. All interaction effects were nonsignificant.

For each S, mean response speeds on Trials 31 through 70 were calculated for each of the four sequential events and submitted to a Linquist (1953) Type VI analysis of variance. The between-Ss variable was stimulus intensity. The SFE comparison (i.e., NS vs FS) and the CE comparison [i.e., change from the previous trial (NS-FS + FS-NS) vs no change

Table 1

Mean Response Speeds to the FS and NS on Change and No Change Trials

\begin{tabular}{lccc} 
& \multicolumn{3}{c}{ Stimulus Event } \\
\cline { 2 - 4 } Change Value & FS & NS & Overall \\
\hline Change & 1.236 & 1.254 & 1.245 \\
No Change & 1.238 & 1.281 & 1.260 \\
\hline Overall & 1.237 & 1.267 & \\
\hline
\end{tabular}


(NS-NS + FS-FS)] were the within-Ss variables. The SFE comparison was significant $(F=8.30, \mathrm{df}=1 / 69, \mathrm{p}<.01)$; that is, response speeds were faster to the NS than to the FS. The stimulus intensity effect $(F=2.40, \mathrm{df}=2 / 69, \mathrm{p}>.10)$ and the $C E$ comparison $(F=2.53, \mathrm{df}=1 / 69, \mathrm{p}>.10)$ were not significant. None of the interactions were significant. Table 1 shows the mean response speeds of interest for the two-stimulus RT phase.

\section{DISCUSSION}

The failure to find the Stimulus Intensity by Trial Block interaction in the familiarization phase and the SFE by Intensity interaction in the two-stimulus RT phase may be attributed to the fact that intensity effects are less pronounced when this variable is employed in a between-Ss rather than in a within-Ss design (Grice \& Hunter, 1964). A second possibility is that the range of stimulus intensity values used in this study may have been too restricted to obtain either of the effects. A third possibility may be that variations in stimulus intensity do not result in differential habituation of children's "attending" responses in simple $\mathrm{RT}$ tasks.

The significant SFE result is consistent with the findings of other investigations (Bogartz \& Witte, 1966; Cantor \& Cantor, 1964, 1965, 1966; Witte, 1967; Witte \& Cantor, 1967). Further inspection of the data indicated that speeds to the NS decreased only from the first to the second trial block, whereas, speeds to the FS decreased over all four trial blocks. Mean trial block speeds to the NS were $1.299,1.247,1.255$, and 1.255 , respectively, while mean trial block speeds to the FS were $1.271,1.248,1.216$, and 1.214 , respectively. Thus, the magnitude of the SFE (i.e., difference in mean speeds to the NS and FS) varied across trial blocks.

The nonsignificant $\mathrm{CE}$ finding is in agreement with the results of a previous study (Bogartz \& Witte, 1966,
Experiment 1) which employed a simple RT task, and also studies which have involved a lever pulling response (e.g. Witte, 1967). Cantor \& Cantor (1966) suggested that the CE may be due to a form of "novelty" inherent in changing from one stimulus to another from trial to trial within the motor task. The data for the CE comparison is inconsistent with this hypothesis.

\section{REFERENCES}

BOGARTZ, $R$, $S$, WITTE, $K$, $L$. On the locus of the stimuhus familiarization effect in young children. Journal of Experimental Child Psychology, 1966, 4, 317-331.

CANTOR, G. N., CANTOR, J. H. Effects of conditioned-stimulus familiarization on instrumental learning in children. Joumal of Experimental Child Psychology, 1964, 1, 71-78.

CANTOR, G. N., \& CANTOR, J. H. Discriminative reaction time performance in children as related to stimulus familiarization. Jouma of Experimental Child Psychology, 1965, 2, 1-9.

CANTOR, G. N., \& CANTOR, J. H. Discriminative reaction time in children as related to amount of stimulus familiarization. Journal of Experimental Child Psycholosy, 1966, 4, 150-157.

GRICE, G. R., \& HUNTER, J. J. Stimulus intensity effects depend upon the type of experimental design. Psychological Review, 1964, 71, 247-256.

LINQUIST, E. F. Design and analysis of experiments in psychology and education. Boston: Houghton-Mifflin, 1953.

THOMPSON, R. F., \& SPENCER, W. A. Habituation: A model phenomenon for the study of neuronal substrates of behavior. Psychological Review, 1966, 73, 16-43.

WITTE, K. L. Children's response speeds to familiarized stimuli. Psychonomic Science, 1967, 7, 153-154. NOTES

1. Funds for conducting this research were provided by a grant from the Graduate School, University of South Dakota.

2. This research was conducted during the second author's tenure as a Public Health Service Trainee, National Institute of Mental Health. The cooperation of Mrs. Hazel Linderman, principal, and Mrs. Virginia Stedronsky, kindergarten teacher, of Austin School, Vermillion, South Dakota, is gratefully acknowledged. 